\title{
Primates and mouse NumtS in the UCSC Genome Browser
}

\author{
Francesco Maria Calabrese, Domenico Simone, Marcella Attimonelli ${ }^{*}$ \\ From Eighth Annual Meeting of the Italian Society of Bioinformatics (BITS) \\ Pisa, Italy. 20-22 June 2011
}

\begin{abstract}
Background: NumtS (Nuclear MiTochondrial Sequences) are mitochondrial DNA sequences that, after stress events involving the mitochondrion, colonized the nuclear genome. Accurate mapping of NumtS avoids contamination during mtDNA PCR amplification, thus supplying reliable bases for detecting false heteroplasmies. In addition, since they commonly populate mammalian genomes (especially primates) and are polymorphic, in terms of presence/ absence and content of SNPs, they may be used as evolutionary markers in intra- and inter-species population analyses.

Results: The need for an exhaustive NumtS annotation led us to produce the Reference Human NumtS compilation, followed, as reported in this paper, by those for chimpanzee, rhesus macaque and mouse ones. Identification of NumtS inside the UCSC Genome Browser and their inter-species comparison required the design and the implementation of NumtS tracks, starting from the compilation data. NumtS retrieval through the UCSC Genome Browser, in the species examined, is now feasible at a glance.

Conclusions: Analyses involving NumtS tracks, together with other genome element tracks publicly available at the UCSC Genome Browser, can provide deep insight into genome evolution and comparative genomics, thus improving studies dealing with the mechanisms that drove the generation of NumtS. In addition, the NumtS tracks constitute a useful tool in the design of mitochondrial DNA primers.
\end{abstract}

\section{Background}

Colonization of the nuclear genome by mitochondrial DNA fragments began shortly after endosymbiosis and, while still ongoing, has laid the groundwork for a scenario composed of recombination events which generated duplications and de novo insertions. The integration or rather the "capture" of these fragments may occur during repair of double-strand breaks in nuclear DNA by

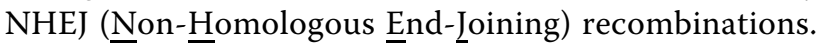
These events arise due to the action of endogenous and exogenous agents such as ionizing radiation and are strictly dependent on the rate of double-strand breaks in nuclear DNA $[1,2]$. In some organisms, particularly primates, the same mitochondrial region occurs several times along the nuclear genome, showing how

\footnotetext{
* Correspondence: m.attimonelli@biologia.uniba.it

Dipartimento di Bioscienze, Biotecnologie e Scienze Farmacologiche, Università di Bari, Bari, 70126, Italy
}

\section{Biomed Central

(c) 2012 Calabrese et al.; licensee BioMed Central Ltd. This is an open access article distributed under the terms of the Creative Commons Attribution License (http://creativecommons.org/licenses/by/2.0), which permits unrestricted use, distribution, and reproduction in any medium, provided the original work is properly cited. duplication events have driven the spread of NumtS among these species [1-3]. This is also indicated by the discrepancies in NumtS content among human and chimpanzee, although they are closely located in the phylogenetic tree. Another important feature of NumtS concerns the intra-specific polymorphic state, both in terms of presence/absence and SNP content: they may occur in homo- or heterozygosis or are absent in various individuals at specific loci. This feature makes them suitable for human and primate population analyses, since the most enriched species harbored this branch of the whole phylogenetic tree $[4,5]$. Although several clinical studies report data on NumtS involvement in causing heteroplasmy artefacts of mtDNA PCR amplification, no works have so far been published reporting tools allowing the NumtS browsing. NumtS content among mammalian and non-mammalian species has also been investigated, but the data published so far do not allow the indepth 
knowledge of the genomic rearrangements in which NumtS are involved. The construction of annotation tracks within the UCSC Genome Browser will certainly improve such investigations. Setting the human "NumtSome" catalog on hg18 build allowed the complete collection of a nuclear-mitochondrial "library" comprising more than 500 NumtS, i.e. RHNumtS.2 [6], and new compilations for other organisms have also been produced. In more detail, the same optimized protocol which allowed the detection of human NumtS, based on in silico hybridization between the human mtDNA reference sequence (rCRS) [7] and the human nuclear genome assembly, was applied to Pan troglodytes and Mus musculus. Our choice fell on those species which, at the time of the inspection (2010), were reported in the NCBI genome statistics pages as mammalian genomes in a complete state of assembly. In order to take into account an outgroup for the hominoid branch, the NumtS annotation in rhesus macaque (Macaca mulatta) was also performed, although its genome was, at the time of the study, in a draft state of assembly. As regards chimpanzee and mouse, although several studies have published the number of NumtS and their genome coverage $[3-5,8,9]$, no data about genome positions are available. Here we report an improvement in terms of the quantity and quality of data and the complete annotations of NumtS. This kind of data for rhesus macaque have never been published. The NumtS compilations produced for the above-mentioned species allowed the design of tracks inside the UCSC Genome Browser. The growing demand for genomic details in these species, which are commonly used within the UCSC, encouraged us to publish the NumtS mouse (mm9 build) and human (hg19 build) tracks. Annotations about NumtS in the species (chimpanzee and rhesus macaque) which, in our opinion, will be extensively used, can be uploaded as NumtS custom annotation tracks from our local server. Our purpose is to provide a proper support to NumtS surveys and to facilitate comparative NumtS analyses, an aspect which has previously been carried out only in chimpanzee/ human comparisons [3-5].

\section{Methods}

Blasting of nuclear genome versus mitochondrial genome Bl2seq, which implements the BlastN program (release 2.2.19 of the Blast suite) thus allowing pairwise comparisons, was launched on the entire chromosomal set for each species: 24 for Pan troglodytes (panTro2 build), 21 for Macaca mulatta (rheMac2 build) and Mus musculus (mm9 build). The entire chromosome dataset for each build was downloaded at the following address [10]. Job executions were performed on a local server. The accession numbers of the mtDNA reference sequences used as queries are respectively $\mathrm{NC}_{-} 005943$ for rhesus macaque, NC_001643 for chimpanzee and NC_005089 for mouse. The same protocol applied to the generation of the human compilation (RHNumtS.2) [6] was used for the other three species, with scoring parameters fixed as follows: 2 for match reward, -3 for mismatch penalty, -5 for gap opening, -2 for gap extension. The expected value (e-value) was fixed at 1e-03. Each fragment of each chromosome aligned with the mtDNA whose e-value was lower than the fixed threshold produced an HSP (High Scoring Pair). Each HSP was associated with a NumtS. The complete list of NumtS loci for each species was annotated in a spreadsheet (NumtS compilation).

\section{NumtS assembly}

NumtS mapping at a distance of less than 2000 bp on a specific chromosome and corresponding to two mtDNA fragments, at most distant $2000 \mathrm{bp}$ and oriented in the same direction, were combined in a single NumtS and called "assembled NumtS". This was done by spreadsheet interpolation and manual inspection, well supported by graphical display on the UCSC Genome Browser and with tools available within the Galaxy platform [11-14]. As in the generation of human assembled NumtS, the fragment-joining protocol was slightly modified for HSPs, interposed by long repetitive elements in order to compare fragments which followed the same evolutionary history, at least among primate lineage.

\section{NumtS track implementation}

Starting from the NumtS compilation for each species, four tracks were implemented: "NumtS" and "NumtS Assembled" tracks, displaying data from the corresponding compilations; the "NumtS on mitochondrion" track, showing mapping of NumtS on the mitochondrial genome, and the "NumtS on mitochondrion with mismatches" track, showing mismatches between NumtS sequence and mitochondrial genome. Since the mitochondrial chromosome is not available in the UCSC Genome Browser for Macaca mulatta, the "NumtS on mitochondrion" and the "NumtS on mitochondrion with mismatches" tracks were not designed for this species.

\section{Creating BED and BAM tracks}

UCSC Genome Browser tracks may be produced and configured in a variety of ways, to highlight features needed. The format used for "NumtS", "NumtS Assembled" and "NumtS on mitochondrion" tracks is the Browser Extensible Data format (BED) which is a flexible way of showing genomic annotations. In-house shells and Python scripts were used to produce BED and BAM formatted data and to implement interNumtS track links, starting from the UCSC description pages, providing further insights when switching from 
the nuclear locus of a NumtS to its mitochondrial counterpart. The BAM format, the compressed binary version of the Sequence Alignment/Map (SAM) format, was used for the "NumtS on mitochondrion with mismatches" track generation. The LASTZ software [15] was used to realign each NumtS to its mitochondrial sequence, using default parameters and setting the SAM format as output. SAM files were converted to BAM format with the SAMtools package [16]. Figure 1 shows the flowchart followed to obtain the NumtS tracks starting from the Blastn, giving HSPs and passing through NumtS compilations.

\section{Remapping of human tracks on hg19}

The revised reference human NumtS compilation (RHNumtS.2), already implemented inside the UCSC Genome Browser according to the hg18 build, was upgraded to the GRCh37 build (hg19). Genomic coordinates were converted by using the "Lift-Over" tool available through the Galaxy suite. Human hg19 compilation is available in additional file 1, RHNumtS.2 compilation.

\section{Comparison with previously published compilations}

To compare NumtS intervals with those available in other previously published NumtS compilations, the "Lift-Over" tool, based on Lift-Over utility and Chain track, and the "Join" tool were used. They are both free

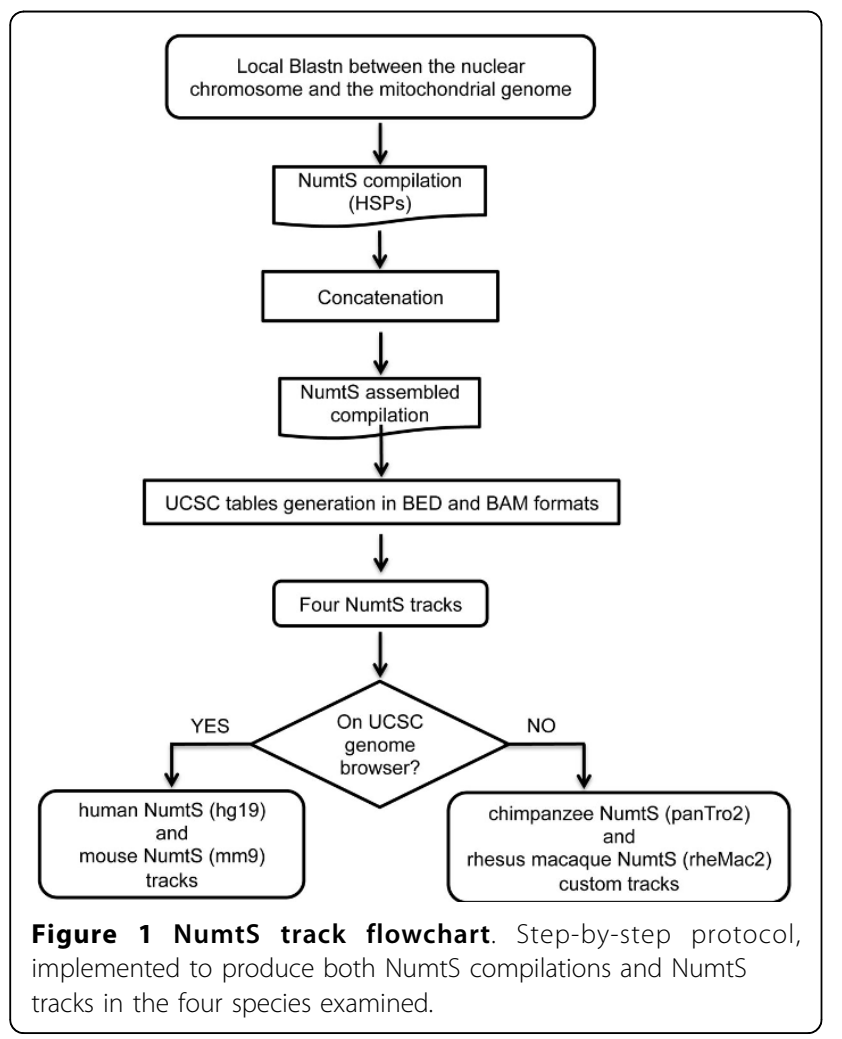

and available in the Galaxy suite at UCSC Genome Browser [14].

\section{Results}

NumtS insertions appear to have been more or less continuous over time in the lineages leading to the human genome [2-5,8]. After the RHNumtS.2, in view of the proximity between human and chimpanzee (5-7 million of years) and taking into account the level of similarity between their genomes, the NumtS comparison between them is an important step in the evolutionary study of the NumtS. Thus, by applying the same protocol used for RHNumtS.2, we have produced respectively the RPNumtS, RRNumtS and RMNumtS compilations for Pan troglodytes, Macaca mulatta and Mus musculus species. Detailed analyses of the quantity of the speciesspecific NumtS insertion/duplication events which occurred after the chimpanzee-human divergence are needed, to understand the underlying phylogenetic relationships. We produced the UCSC tracks with this aim in mind.

\section{Gathering NumtS in Pan troglodytes}

The RPNumtS (Reference Pan troglodytes NumtS) compilation (additional file 2, RPNumtS compilation) annotates 776 NumtS, including 117 NumtS assembled by applying the criteria described in the Methods section. The nuclear spans range from $33 \mathrm{bp}$ to $8984 \mathrm{bp}$, with a percentage of similarity ranging from 64.4 to 100 (Figure 2). An ID was assigned to each chimpanzee NumtS according to the format Ptr_NumtS_xxx, where Ptr stands for Pan troglodytes and $\mathrm{xxx}$ is a three-digit code.

\section{Gathering NumtS in Macaca mulatta}

The number of HSPs returned by the similarity search analysis in rhesus macaque was 751, 113 of which were concatenated. The nuclear spans range from 28 bp to $6642 \mathrm{bp}$ with a percentage of similarity ranging from 64 to 100 (Figure 2). The ID format used for the rhesus macaque NumtS was Rhm_NumtS_xxx, where Rhm stands for rhesus macaque. The RRNumtS (Reference Rhesus NumtS) compilation is available in additional file 3, RRNumtS compilation.

\section{Gathering NumtS in Mus musculus}

The number of HSPs returned by the Blastn on the mouse genome is 172 of which 13 were assembled. The nuclear spans range from $33 \mathrm{bp}$ to $4654 \mathrm{bp}$ with a percentage of similarity ranging from 66 to 100 (Figure 2). Also for mouse the nomenclature follows the same rule as the other species: Mms_NumtS_xxx where Mms stands for Mus musculus. The RMNumtS (Reference Mouse NumtS) compilation is available in additional file 4, RMNumtS compilation. 


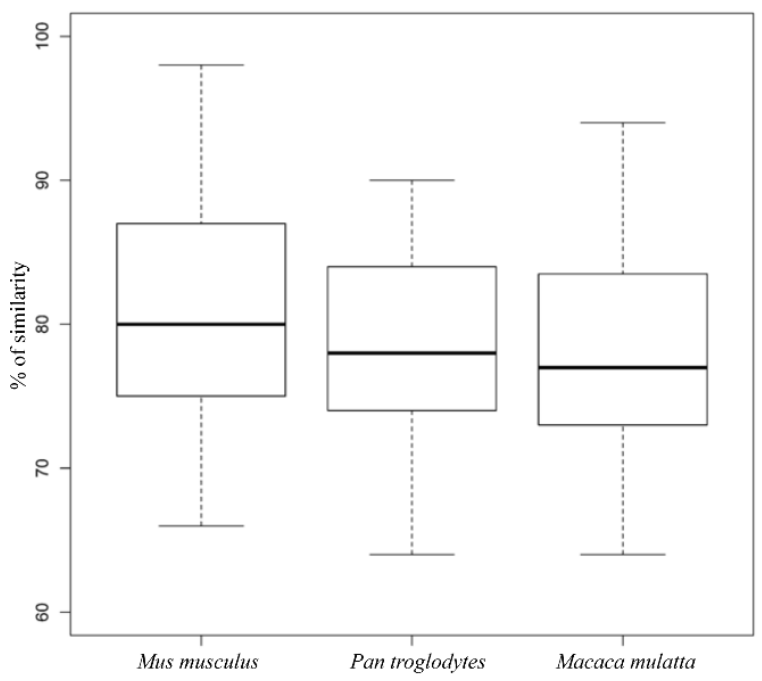

b

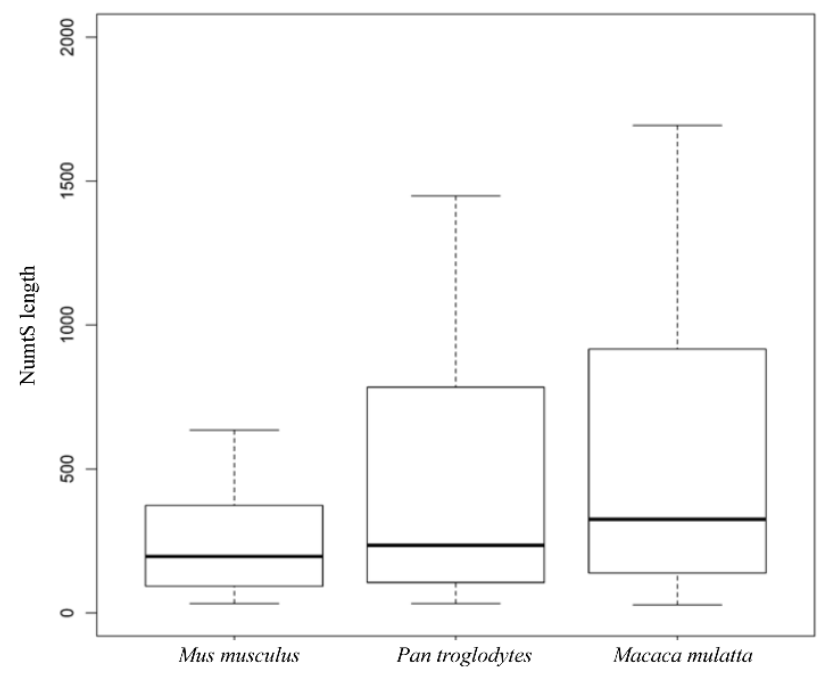

Figure 2 Comparison of the box plots for NumtS lengths and similarity distributions among species examined. a) Box plot based on distribution of NumtS percentage of similarity with respect to corresponding mitochondrial reference genome in three species examined. Mus musculus has the highest values. b) Box plots for Numts lengths, based on mouse, chimpanzee and rhesus macaque compilations. Rhesus macaque shows highest median and maximum values.

\section{Browsing the NumtS tracks inside the UCSC Genome Browser}

Data in additional files 1, 2, 3 and 4 were used to set up the "NumtS Sequence" track group on the UCSC Genome Browser ("Variation and Repeats" section) for human on hg19, chimpanzee, rhesus macaque and mouse, respectively. Implementation of the NumtS compilations on the UCSC Genome Browser for human and mouse data facilitates access to the NumtSome annotations. Once connected to UCSC [17], the presence of any NumtS in a human (hg18 or hg19 build) or murine (mm9) genomic region of interest (selected by typing its coordinates in the "position/term" search box) can be checked; alternatively, by typing a NumtS identifier in the same search box, a list of tracks available for that NumtS appears. Clicking on one of the results displays the selected item in the Genome Browser. For each NumtS, the description page reports a link which guides users in the shift from the NumtS nuclear position to its mitochondrial counterpart and vice versa. Because tracks can easily connect annotation data, they provide a very useful tool in understanding the evolutionary dynamics in which NumtS are involved and characterizing their genomic context. Pan troglodytes NumtS tracks, available on our local server, can be uploaded as custom annotation tracks starting from the "NumtS on mitochondrion track" with the link reported in the reference section [18]. Starting from the mitochondrial track, clicking on any NumtS shows external page providing further specifications about that NumtS (together with the others) in
UCSC Genome Browser "detail page" fashion. This procedure substitutes the "NumtS Sequence" track activation described above for human and mouse tracks. For M. mulatta custom tracks activation, because of the lack in the rhesus macaque build of the mitochondrial genome, means that the starting link points to a nuclear location, at the same time activating the NumtS track. The link is reported in the reference section [19].

\section{Comparisons with previously published compilations}

Other research groups have already reported NumtS overviews in primates and mouse. In order to demonstrate the improvements obtained with our approach, comparisons between our compilations and publicly available data for chimpanzee $[3,5,9]$, rhesus macaque $[3,9]$ and mouse $[8,9]$ are reported here. The results are listed in additional file 5 , NumtS compilations comparison. HSP number and base pair coverage on the entire genome, as well as the same features for the assembled NumtS, are given. Differences in compilation contents for the same organism are due to differences between one release and another and to the Blastn algorithm parameters chosen. The only NumtS locations available are those published in [5] concerning NumtS in Pan troglodytes. Merging of these annotations with our compilation shows that all the items reported in [5] are included within our dataset. Further details about the comparisons of NumtS compilations are given in additional file 6, RPNumtS annotation comparison. 


\section{Discussion and conclusions}

The above protocol produced 776, 751 and 173 HSPs in chimpanzee, rhesus macaque and mouse, respectively. These data confirm and extend the NumtS catalog for different species, published so far and summarised in [8]. To test the statistical significance of the NumtS lengths in each species, we have used a Pearson correlation matrix (additional file 7, NumtS and genome features

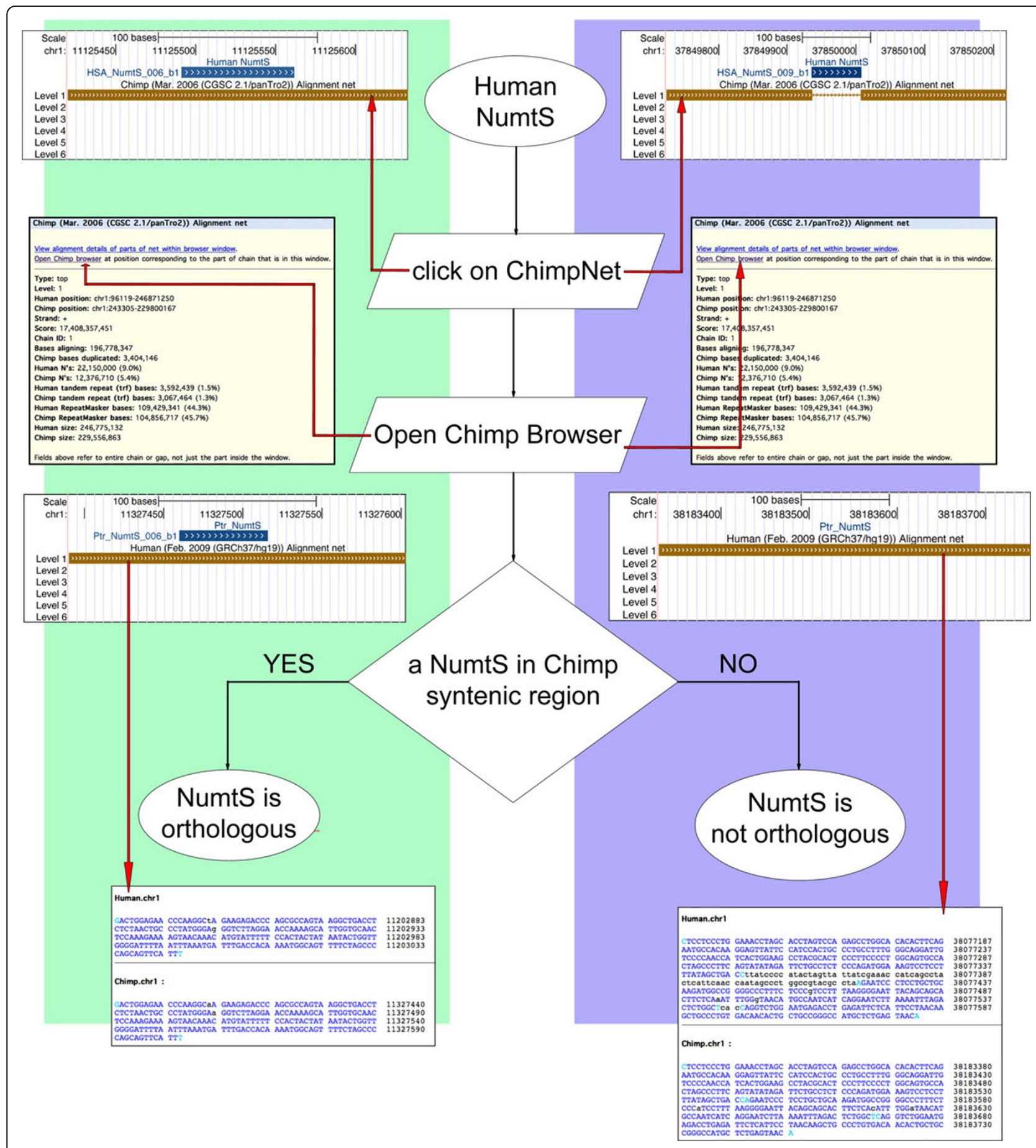

Figure 3 Browsing UCSC NumtS tracks to identify orthologous NumtS. Workflow shown assesses presence of identical NumtS in two species for which the UCSC annotation tracks were produced. Graphs show two human NumtS, (HSA_NumtS_006_b1, HSA_NumtS_009_b1) and ChimpNet track, indicating that the former is also present in chimpanzee, whereas the latter is not. Following flowchart (and clicking on links and boxes marked with red arrows), this hypothesis can be verified on Chimp Browser. Alignment provided by Net tracks provides additional evidence of presence or absence of NumtS in species examined. See additional file 8, Figure 2 screenshots for magnified versions of screenshots. 


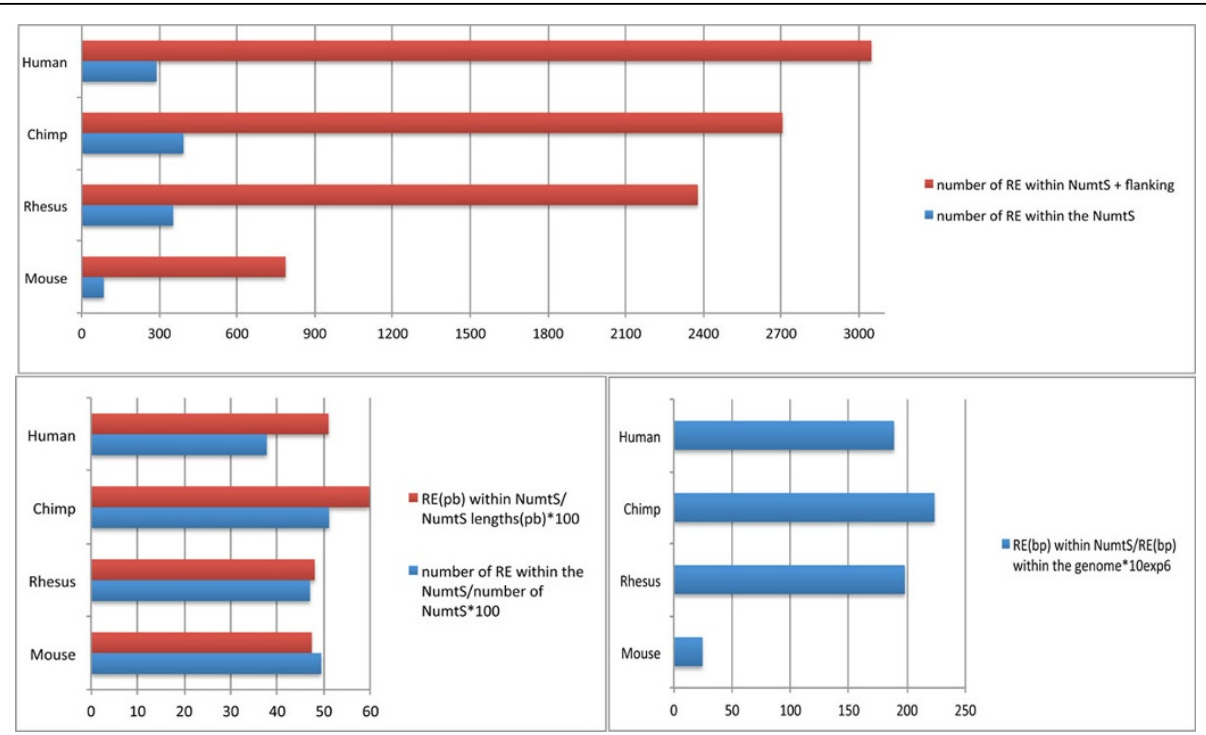

Figure 4 Repeated elements within NumtS: content comparisons among species examined. a) Total amount of RE within NumtS genomic intervals, with and without flanking regions (1000 bp upstream and downstream), calculated for each species. Trend in any of species examined reveals more REs in flanking regions with respect to NumtS region alone, with greater emphasis on mouse genome. b) Total RE content (bp and number) in NumtS loci on total NumtS length and number respectively, calculated as percentage. c) Percentage of RE(bp) within NumtS loci on RE content within whole genome. Although mouse genome is less populated with REs, its NumtS are populated by REs with same percentage observed in other species examined.

Table 1 Chimpanzee NumtS annotation as reported in the compilation limited to the mitochondrial region 1006-1771 considered in the Primer selection example

\begin{tabular}{|c|c|c|c|c|c|}
\hline \#chrom & chromStart & chromEnd & NumtS_ID & score & strand \\
\hline chrM & 318 & 2790 & Ptr_NumtS_376_b1 & 983 & + \\
\hline chrM & 0 & 2116 & Ptr_NumtS_443_b1 & 951 & - \\
\hline chrM & 457 & 2391 & Ptr_NumtS_442_b1 & 944 & - \\
\hline chrM & 0 & 2512 & Ptr_NumtS_379_b2 & 840 & + \\
\hline chrM & 0 & 2512 & Ptr_NumtS_315_b2 & 838 & + \\
\hline chrM & 0 & 2831 & Ptr_NumtS_667_b2 & 808 & - \\
\hline chrM & 835 & 2448 & Ptr_NumtS_127_b1 & 805 & + \\
\hline chrM & 0 & 2586 & Ptr_NumtS_192_b2 & 794 & + \\
\hline chrM & 19 & 2227 & Ptr_NumtS_106_b2 & 790 & - \\
\hline chrM & 703 & 2783 & Ptr_NumtS_371_b1 & 790 & + \\
\hline chrM & 21 & 5619 & Ptr_NumtS_039_b1 & 786 & - \\
\hline chrM & 79 & 2939 & Ptr_NumtS_343_b2 & 785 & - \\
\hline chrM & 0 & 2249 & Ptr_NumtS_604_b1 & 779 & - \\
\hline chrM & 0 & 2236 & Ptr_NumtS_423_b1 & 775 & + \\
\hline chrM & 19 & 5841 & Ptr_NumtS_073_b2 & 774 & + \\
\hline chrM & 4 & 4498 & Ptr_NumtS_335_b2 & 770 & + \\
\hline chrM & 84 & 9112 & Ptr_NumtS_194_b2 & 770 & - \\
\hline chrM & 0 & 1786 & Ptr_NumtS_516_b1 & 767 & - \\
\hline chrM & 0 & 4603 & Ptr_NumtS_088_b1 & 765 & - \\
\hline chrM & 0 & 4321 & Ptr_NumtS_699_b2 & 758 & + \\
\hline chrM & 0 & 4343 & Ptr_NumtS_698_b1 & 758 & - \\
\hline chrM & 0 & 4343 & Ptr_NumtS_685_b1 & 758 & - \\
\hline chrM & 0 & 4321 & Ptr_NumtS_697_b2 & 757 & + \\
\hline chrM & 0 & 4321 & Ptr_NumtS_693_b1 & 757 & - \\
\hline chrM & 0 & 4343 & Ptr_NumtS_700_b1 & 756 & - \\
\hline
\end{tabular}


Table 1 Chimpanzee NumtS annotation as reported in the compilation limited to the mitochondrial region 1006-1771 considered in the Primer selection example (Continued)

\begin{tabular}{lllllc}
\hline chrM & 0 & 4321 & Ptr_NumtS_695_b1 & 756 & - \\
chrM & 0 & 4321 & Ptr_NumtS_694_b2 & 756 & + \\
chrM & 0 & 4343 & Ptr_NumtS_692_b2 & 756 & + \\
chrM & 0 & 4343 & Ptr_NumtS_691_b1 & 756 & - \\
chrM & 0 & 4321 & Ptr_NumtS_690_b2 & 756 & + \\
chrM & 0 & 4343 & Ptr_NumtS_687_b1 & 756 & - \\
chrM & 0 & 4343 & Ptr_NumtS_623_b3 & 755 & + \\
chrM & 19 & 4343 & Ptr_NumtS_686_b3 & 746 & + \\
chrM & 19 & 4343 & Ptr_NumtS_696_b1 & 745 & + \\
chrM & 19 & 4343 & Ptr_NumtS_684_b1 & 745 & + \\
chrM & 0 & 2183 & & + \\
\hline
\end{tabular}

correlation matrix), highlighting a strong correlation between genome size and total amount of NumtS. Figures $2 \mathrm{a}$ and $2 \mathrm{~b}$ show that mouse NumtS are the least, the shortest but also the most highly conserved. The availability of the tracks will allow us to go into further detail on this aspect. In order to facilitate NumtS inter- and intra-species studies on the basis of our compilations, we produced the UCSC Genome Browser tracks, representing a reliable and useful way of recognizing and interrelating them with other genomic elements. Among the most important advantages linked to track implementation are i) the comparison of NumtS data with any other type of genomic element annotation through tracks in the browser, thus allowing NumtS to be mapped in syntenic regions as well as in repetitive regions; ii) the possibility of retrieving the entire set of a track data

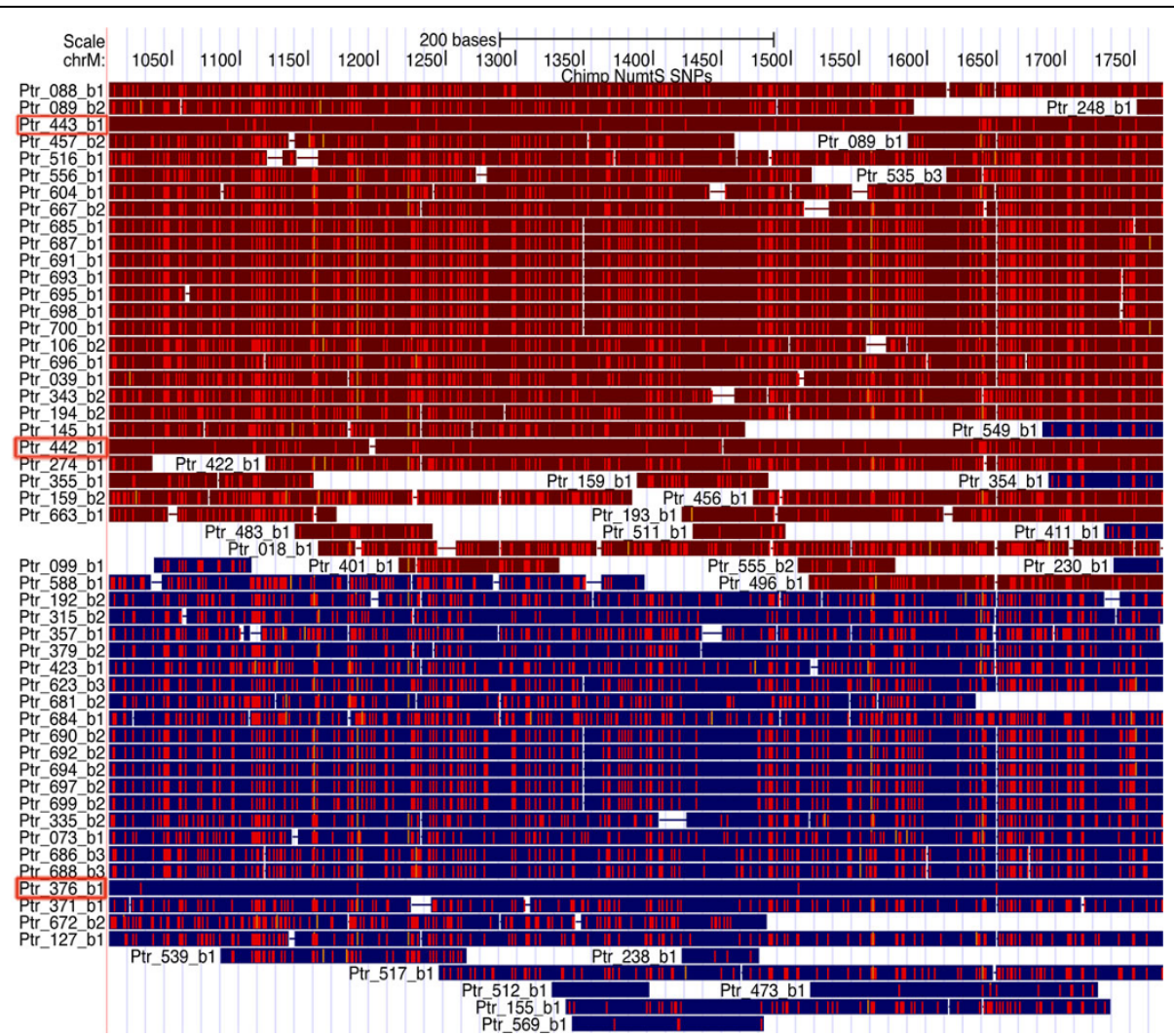

Figure 5 Evidence of risk of coamplification due to NumtS. Chimpanzee NumtS spanning within mitochondrial region from 1006 to 1771 ; first and second large blocks in red and blue include NumtS on minus and plus strand respectively. Red squared NumtS IDs (Ptr_NumtS _443_b1 and Ptr_NumtS_376_b1) correspond to potentially unintended nuclear regions found by Primer-Blast software, and have fewest mismatches. Red squared NumtS Ptr_NumtS_442_b1 not found by Primer-Blast software, has more mismatches. 
through the "Tables" section, allowing a custom NumtS database to be produced; iii) the possibility of checking for risk of co-amplification in the process of mtDNA amplification [20].

\section{Syntenic analyses}

The whole UCSC Genome Browser is in fact a container of browsers for various species. It can be used to classify the NumtS of two different species as orthologous or not simply by using genome browsers for which NumtS tracks have been designed and implemented. Starting from a NumtS in one of the species, for which data in the comparative genomic section are available, and shifting to another genome browser for which the NumtS tracks have also been set up, the presence/absence of a gap in the NumtS region can identify species-specific or orthologous NumtS. As an example to demonstrate the advantage of the availability of NumtS tracks for different species, starting from the HSA_NumtS_006 in the human browser, Figure 3 shows the path to be followed to recognize its orthologous, Ptr_NumtS_006 in chimpanzee (for magnified screenshots of Figure 3, see additional file 8, Figure 2 screenshots).

\section{NumtS and RE content among primates and mouse genomes}

Figure 4 shows the statistical estimate of the relationship between NumtS and Repetitive Elements (RE). In all the considered species, as the number of RE in the regions, including the flanking region, is significantly high, NumtS insertions may occur preferentially in highly repetitive regions, with a bias in human NumtS, taking into account the fact that the chimpanzee genome has a greater quantity of NumtS. A more detailed analysis in terms of length of both NumtS and RE indicates that chimpanzee NumtS contain a higher density of RE with respect to the others. This is confirmed by the trend of the ratio between the base coverage of $\mathrm{RE}$ within NumtS with respect to the same value found in the entire genome (Figures $4 \mathrm{~b}$ and $4 \mathrm{c}$ ).

\section{Risk of co-amplification}

As a result of finding pair-specific PCR primer for a chimpanzee mitochondrial region (e.g. range 10001800 in the mitochondrial genome), the Primer-Blast software [21] returns two products on potentially unintended nuclear templates for the best primer pair. Browsing the NumtS tracks proves that the two nuclear regions each contain a NumtS. Table 1 lists the NumtS sorted by score, which spans the same chimpanzee mitochondrial region, extracted from the "Ptr_NumtS on mitochondrion" track in the relative table browser. The first two highest scores are for
Ptr_NumtS_376_b1 and Ptr_NumtS_443_b1, as shown in Figure 5. The third score value corresponds to Ptr_NumtS_442_b1, which contains more differences than the other two with respect to hg18 (and is thus not considered in the Primer_Blast report). The match between the data demonstrates the advantage offered by the NumtS tracks in avoiding NumtS coamplification.

To conclude, as demonstrated in the above discussion, the compilations reported here represent a complete set of primate and mouse NumtS loci.

\section{Additional material}

Additional file 1: RHNumtS.2 compilation. Human reference NumtS compilation on hg19 build. Each line refers to a NumtS and reports NumtS ID, HSP_NumtS ID, chromosome where NumtS is located, and strand where Blast mapped NumtS ("+" if in same direction as nuclear reference sequence, "-" if in opposite direction), chromosome and mitochondrial locations. For assembled NumtS (right), detailed information on each fragment is available. Last column shows identity percentage as reported in Blast output for each HSP.

Additional file 2: RPNumtS compilation. Chimpanzee reference NumtS compilation on panTro2 build. Each line refers to a NumtS and gives NumtS ID, HSP_NumtS ID, chromosome where NumtS is located and strand where Blast mapped NumtS ("+" if in same direction as nuclear reference sequence, "-" if in opposite direction), chromosome and mitochondrial locations. For assembled NumtS (right), detailed information on each fragment is available. Last column shows identity percentage as reported in Blast output for each HSP.

Additional file 3: RRNumtS compilation. Macaca mulatta reference NumtS compilation on rheMac2 build. Each line refers to a NumtS and gives NumtS ID, HSP_NumtS ID, chromosome where NumtS is located and strand where Blast mapped NumtS ("+" if in same direction as nuclear reference sequence, "-" if in opposite direction), chromosome and mitochondrial locations. For assembled NumtS (right), detailed information on each fragment is available. Last column shows identity percentage as reported in Blast output for each HSP.

Additional file 4: RMNumtS compilation. Mus musculus reference NumtS compilation on $\mathrm{mm} 9$ build. Each line refers to a NumtS and gives NumtS ID, HSP_NumtS ID, chromosome where the NumtS is located and strand where Blast mapped NumtS ("+" if in same direction as nuclear reference sequence, "-" if in opposite direction), chromosome and mitochondrial locations. For assembled NumtS (right), detailed information on each fragment is available. Last column shows identity percentage as reported in Blast output for each HSP.

Additional file 5: NumtS compilation comparisons. Differences in NumtS number and length (bp) in same species are due to different assembly and differences in parameters used to launch in silico hybridization. Discrepancies observed in both chimpanzee and rhesus macaque data between our results and those reported in [3], where the same assemblies and same e-value were used, cannot be explained, because information on Blast running in [3] is not complete. Instead, trend of differences observed in mouse match assembly time and parameters.

Additional file 6: RPNumtS annotation comparisons. Comparison were made by applying "Join" tool (UCSC Genome Browser) to compare our NumtS genomic intervals with those extracted from only previously published NumtS set available. Since this starting dataset contains orthologous NumtS only (human/chimpanzee orthologous), resulting output is partial. Data reported in [5] exactly match our data.

Additional file 7: NumtS and genome features correlation matrix. A Pearson correlation matrix was calculated to test significance of NumtS lengths in species examined, with respect to relative genome lengths. 
Additional file 8: Figure $\mathbf{2}$ screenshots. This file provides magnified versions of screenshots shown in Figure 2.

\section{List of abbreviations used}

BAM: Binary Alignment/Map; BED: Browser Extensible Data; BI2seq: BLAST 2 sequences; e-value: expected value; HSP: High Scoring Pairs; Mms: Mus musculus; NHEJ: Non-Homologous End-Joining; NumtS: Nuclear mitochondrial Sequences; Ptr: Pan troglōdytes; rCRS: revised Cambridge Reference Sequence; RE: Repeated Elements; Rhm: Rhesus macaque; RHNumtS: Reference Human NumtS (compilation); RMNumtS: Reference Mus musculus NumtS (compilation); RPNumtS: Reference Pan troglodytes NumtS (compilation); RRNumtS: Reference Rhesus macaque NumtS (compilation); SAM: Sequence Alignment/Map.

\section{Acknowledgements}

We are grateful to G. Pesole and E. Picardi for providing access to the PesoleLab server where most analyses were carried out; and A. Zweig and C. Li of the UCSC tracks annotation staff, who allowed us to publish the NumtS tracks at the UCSC Genome Browser.

This work was supported by "Fondo di Ateneo" (University of Bari). This article has been published as part of BMC Bioinformatics Volume 13 Supplement 4, 2012: Italian Society of Bioinformatics (BITS): Annual Meeting 2011. The full contents of the supplement are available online at http:// www.biomedcentral.com/1471-2105/13/S4.

\section{Authors' contributions}

FMC carried out bioinformatics analyses and wrote the manuscript, DS and FMC designed and implemented UCSC tracks in collaboration with the UCSC staff; MA coordinated and supervised the whole project. All authors read and approved the final manuscript.

\section{Competing interests}

The authors declare that they have no competing interests.

Published: 28 March 2012

\section{References}

1. Gaziev Al, Shaǐkhaev GO: Nuclear mitochondrial pseudogenes. Mol Biol (Mosk) 2010, 44(3):405-417.

2. Hazkani-Covo E, Covo S: Numt-mediated double-strand break repair mitigates deletions during primate genome evolution. PLoS Genet 2008, 4(10):e1000834.

3. Hazkani-Covo E: Mitochondrial insertions into primate nuclear genomes suggest the use of numts as a tool for phylogeny. Mol Biol Evol 2009, 26(10):2175-2179.

4. Jensen-Seaman MI, Wildschutte JH, Soto-Calderón ID, Anthony NM: A comparative approach shows differences in patterns of numt insertion during hominoid evolution. J Mol Evol 2009, 68(6):688-699.

5. Hazkani-Covo E, Graur D: A comparative analysis of numt evolution in human and chimpanzee. Mol Biol Evol 2007, 24(1):13-18.

6. Simone D, Calabrese FM, Lang M, Gasparre G, Attimonelli M: The reference human nuclear mitochondrial sequences compilation validated and implemented on the UCSC genome browser. BMC Genomics 2011, 12(1):517.

7. Andrews RM, Kubacka I, Chinnery PF, Lightowlers RN, Turnbull DM, Neil Howell: Reanalysis and revision of the Cambridge reference sequence for human mitochondrial DNA. Nat Genet 1999, 23:147.

8. Hazkani-Covo E, Zeller RM, Martin W: Molecular poltergeists: mitochondrial DNA copies (numts) in sequenced nuclear genomes. PLoS Genet 2010, 6(2):e1000834.

9. Richly E, Leister D: NUMTs in sequenced eukaryotic genomes. Mol Biol Evol 2004, 21(6):1081-1084.

10. UCSC Chromosomes Download Page. [ftp://hgdownload.cse.ucsc.edu/ goldenPath/].

11. Goecks J, Nekrutenko A, Taylor J, Galaxy Team: Galaxy: a comprehensive approach for supporting accessible, reproducible, and transparent computational research in the life sciences. Genome Biol 2010, 11(8):R86.
12. Blankenberg D, Von Kuster G, Coraor N, Ananda G, Lazarus R, Mangan M, Nekrutenko A, Taylor J: Galaxy: a web-based genome analysis tool for experimentalists. Current Protocols in Molecular Biology 2010, Chapter 19(Unit 19.10):1-21.

13. Giardine B, Riemer $C$, Hardison RC, Burhans R, Elnitski L, Shah P, Zhang $Y$, Blankenberg D, Albert I, Taylor J, Miller W, Kent WJ, Nekrutenko A: Galaxy: a platform for interactive large-scale genome analysis. Genome Res 2005, 15(10):1451-1455.

14. Kent WJ, Sugnet CW, Furey TS, Roskin KM, Pringle TH, Zahler AM, Haussler D: The human genome browser at UCSC. Genome Res 2002, 12(6):996-1006.

15. Schwartz S, Kent WJ, Smit A, Zhang Z, Baertsch R, Hardison RC, Haussler D, Miller W: Human-Mouse Alignments with BLASTZ. Genome Res 2003, 13(1):103-107.

16. Li H, Handsaker B, Wysoker A, Fennell T, Ruan J, Homer N, Marth G, Abecasis G, Durbin R, 1000 Genome Project Data Processing Subgroup: The Sequence Alignment/Map (SAM) format and SAMtools. Bioinformatics 2009, 25:2078-2079.

17. UCSC Genome Browser Homepage. [http://genome.ucsc.edu/].

18. Chimpanzee Custom Tracks. [http://genome.ucsc.edu/cgi-bin/hgTracks? $\mathrm{db}=$ panTro2\&position=chrM\&hgt.customText=http://dl.dropbox.com/u/ 31015858/all_PTR_tracks.txt].

19. Rhesus Macaque Custom Tracks. [http://genome.ucsc.edu/cgi-bin/ hgTracks?db=rheMac2\&position=chr1:5484600-5484695\&hgt. customText=http://dl.dropbox.com/u/31015858/all_MMU_tracks.txt].

20. Calvignac S, Konecny L, Malard F, Douady CJ: Preventing the pollution of mitochondrial datasets with nuclear mitochondrial paralogs (numts). Mitochondrion 2011, 11(2):246-254.

21. Primer-Blast Software. [http://www.ncbi.nlm.nih.gov/tools/primer-blast/].

doi:10.1186/1471-2105-13-S4-S15

Cite this article as: Calabrese et al:: Primates and mouse NumtS in the UCSC Genome Browser. BMC Bioinformatics 2012 13(Suppl 4):S15.

\section{Submit your next manuscript to BioMed Central and take full advantage of:}

- Convenient online submission

- Thorough peer review

- No space constraints or color figure charges

- Immediate publication on acceptance

- Inclusion in PubMed, CAS, Scopus and Google Scholar

- Research which is freely available for redistribution

Submit your manuscript at www.biomedcentral.com/submit
C Biomed Central 